\title{
Agenesia vesicular y colangitis, una asociación infrecuente: reporte de caso
}

\author{
Vesicular agenesis and cholangitis, an infrequent association. Case report
}

Agustín González-Covarrubias*, Irais P. Pineda-Tapia, José D. Becerril-Hernández y Manuel Gil-Vargas

Servicio de Cirugía, Hospital General de Puebla Eduardo Vázquez N, Programa Cirugía General Benemérita Universidad Autónoma de Puebla, Puebla, México

\section{Resumen}

Antecedentes: La agenesia vesicular es una malformación congénita poco frecuente. Caso clínico: Varón de 65 años con diagnóstico preoperatorio de agenesia vesicular, cursando con colangitis, colangiopancreatografía retrógrada endoscópica insatisfactoria, resolución por laparotomía, exploración y drenaje biliar. Discusión: La asociación de colangitis y agenesia vesicular ha sido poco reportada en la literatura. Un diagnóstico preoperatorio disminuye el riesgo quirúrgico. La alteración anatómica representa un riesgo de lesión iatrogénica durante la exploración quirúrgica. Conclusiones: La agenesia vesicular es una afección rara, y aún más infrecuente es su asociación con colangitis. Su diagnóstico preoperatorio facilita un plan quirúrgico y evita lesiones iatrogénicas.

Palabras clave: Agenesia vesicular. Coledocolitiasis. Colangitis.

\section{Abstract}

Background: Vesicular agenesis is a rare congenital malformation. Clinical case: Male, 65 years old, preoperative diagnosis of gallbladder agenesis, presenting with cholangitis, unsatisfactory ERCP, resolution by laparotomy, drainage and biliary exploration. Discussion: The association between cholangitis and gallbladder agenesis presents few reports in the universal literature, a preoperative diagnosis reduces surgical risk, anatomical alteration represents an increased risk of iatrogenic injury during surgical exploration. Conclusions: Vesicular agenesis, a rare and even more infrequent entity, its association with cholangitis, its preoperative diagnosis, facilitates a surgical plan avoiding iatrogenic injuries.

Key words: Agenesis. Gallbladder. Choledocholithiasis. Cholangitis.

\section{Introducción}

La agenesia de la vesícula biliar es una anomalía congénita bien reconocida, pero extremadamente rara. Fue descrita por primera vez en 1701 por Lemery, y hasta la fecha se han reportado unos 430 casos en la literatura'.

Existen informes de asociación familiar, favoreciendo la detección entre miembros de la familia asintomáti$\cos ^{2}$. La agenesia aislada es menudo asintomática, pero uno de cada cuatro pacientes presenta dolor

\section{Correspondencia:}

*Agustín González-Covarrubias

Antiguo camino a Guadalupe Hidalgo 11350

Col. Agua Santa

Fecha de recepción: 15-03-2021

C.P. 72490 , Puebla, Pue., México

E-mail: guty_x@ hotmail.com

0009-7411/@ 2021 Academia Mexicana de Cirugía. Publicado por Permanyer. Este es un artículo open access bajo la licencia CC BY-NC-ND (http://creativecommons.org/licenses/by-nc-nd/4.0/). 
abdominal superior derecho, náuseas e intolerancia a los alimentos grasos ${ }^{3}$. La agenesia de la vesícula biliar a menudo se asocia con estasis en las vías biliares, lo que produce características clínicas de colecistitis, colangitis y coledocolitiasis ${ }^{3}$.

La importancia de realizar un diagnóstico preoperatorio radica en que se minimiza el riesgo quirúrgico, debido a que la ausencia de estructuras anatómicas normales y la incapacidad de manipular la vesícula biliar para disecar el triángulo de Calot representan un riesgo de lesión iatrogénica ${ }^{4}$.

\section{Caso clínico}

Varón de 65 años, sin antecedes médicos de importancia, sin cirugía abdominal previa, que acude al servicio de urgencias con un cuadro de ictericia progresiva de 4 días de evolución, al que posteriormente se agrega dolor en el hipocondrio derecho de tipo cólico, náusea, vomito de contenido gastrobiliar y fiebre. Es hospitalizado y se reportan los siguientes parámetros de laboratorio: leucocitos 28.3 , neutrófilos 92.9\%, bandas 24, plaquetas (PLT) 121, hemoglobina (HB) 16.1, urea 128, nitrógeno ureico en sangre 60, bilirrubina total $7 \mathrm{mg} / \mathrm{dl}$, bilirrubina directa $5.2 \mathrm{mg} / \mathrm{dl}$, bilirrubina indirecta $1.8 \mathrm{mg} / \mathrm{dl}$, fosfatasa alcalina $350 \mathrm{mg} / \mathrm{dll}$, gamma-glutamil transferasa $258 \mathrm{UI}$. Tiempos de coagulación: tiempo de tromboplastina parcial 32.2, tiempo de protrombina 19.9, International Normalized Ratio (INR) 1.6.

Por ultrasonografía de hígado y de vías biliares con transductor convexo se concluye incapacidad para visualizar la vesícula biliar por probable interposición de asa, con dilatación de la vía biliar intrahepática y extrahepática. Posteriormente el paciente es ingresado al servicio de cirugía general con diagnóstico de colangitis moderada secundaria a coledocolitiasis. Como primera conducta se realiza colangiografía por resonancia magnética con un equipo de 1.5 T, multisecuencia (T1, T2, FS, TRUFI, HASTE), de abdomen superior, en la que no se logra delimitar el conducto cístico, el conducto colédoco muestra disminución de su calibre a nivel del segmento intrapancreático, ausencia de señal en la confluencia del conducto pancreático principal e imagen redonda de $6 \mathrm{~mm}$ en el colédoco correspondiente a un lito, que condiciona el paso filiforme de contenido biliar hacia el duodeno, y ausencia de la vesícula biliar en su topografía habitual (Figs. 1-2). El paciente evoluciona con datos de falla renal aguda, oliguria con elevación de azoados y deterioro hemodinámico,

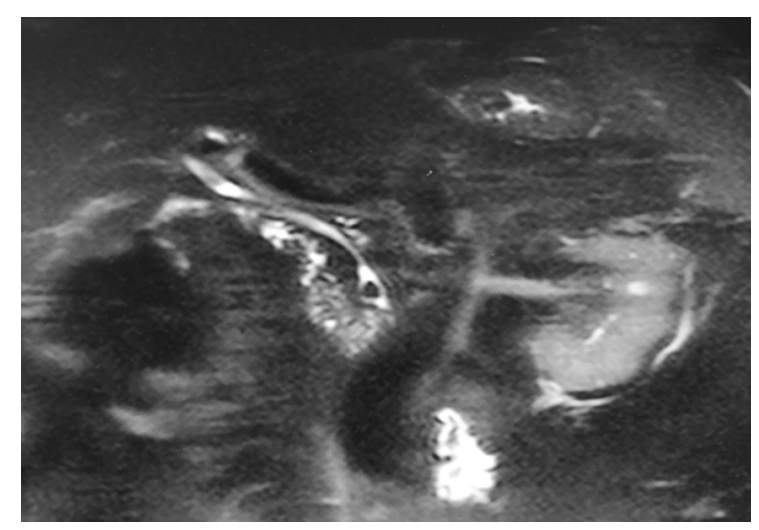

Figura 1. Resonancia magnética que muestra la ausencia de vesícula biliar y un lito en la vía biliar principal.

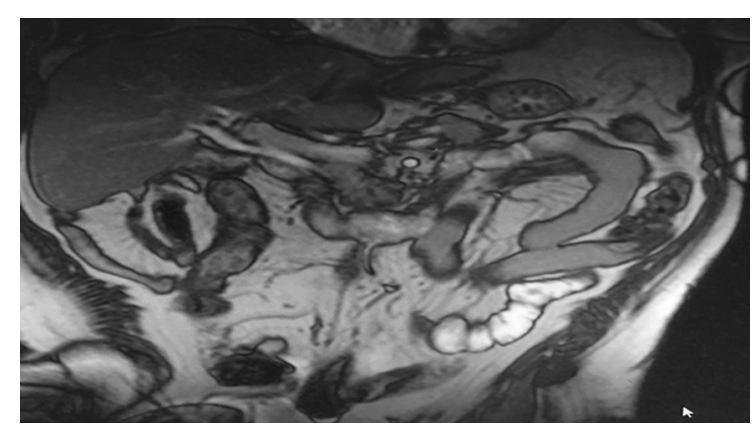

Figura 2. Resonancia magnética que muestra un lito en el segmento intraduodenal del colédoco.

y sepsis con criterios de gravedad secundaria a colangitis moderada condicionada por un proceso obstructivo biliar.

Se realiza colangiopancreatografia retrógrada endoscópica (CPRE) urgente, la cual resulta no exitosa debido a sangrado durante la canulación del colédoco, por lo que se da por terminado el procedimiento. Se decide realizar drenaje de la vía biliar por laparotomía, con exploración de la vía biliar transcoledociana, y extracción de litos sin complicaciones. Como hallazgos quirúrgicos se reportan ausencia de vesícula biliar, con dilatación de colédoco y lito en la porción intraduodenal.

Se realiza colangiografía transoperatoria, que muestra la extracción exitosa de los litos, y se confirma la agenesia vesicular (Fig. 3), hallazgo esperado y concordante con los estudios prequirúrgicos (Figs. 1-2).

El paciente tiene una evolución posquirúrgica favorable, con remisión de los datos de falla orgánica, y es egresado a los 5 días de la cirugía. 


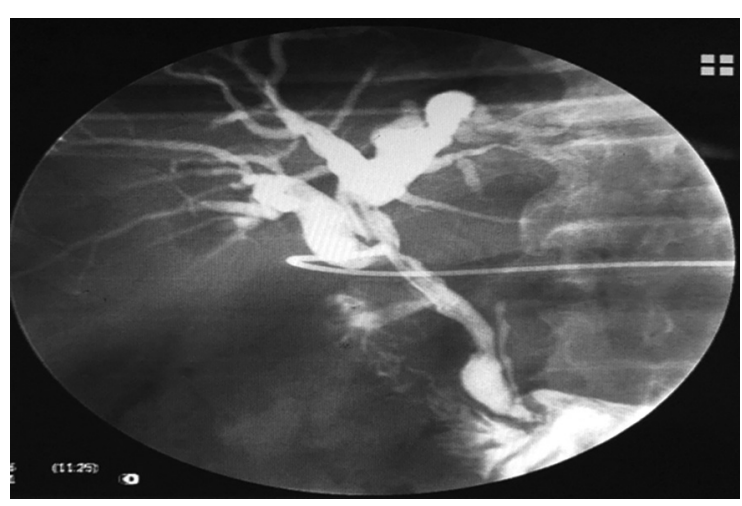

Figura 3. Colangiografía sin defectos de llenado y sin presencia de vesícula biliar.

\section{Discusión}

La agenesia de la vesícula biliar es una aberración embriológica con una incidencia que varía entre el 0,01\% y el 0,075\% (10-75 por 100,000 habitantes) $)^{5}$. La incidencia notificada basada en los hallazgos de autopsias es de aproximadamente un caso por cada 6334 nacidos vivos, o aproximadamente del $0,035 \%$ al $0,3 \%$.

Las mujeres se ven afectadas con mayor frecuencia (3:1) y suelen ser diagnosticadas en la segunda o la tercera década de la vida ${ }^{6}$.

Dentro de la etiopatogenia se describe, durante la semana 3 o 4 de gestación, el sistema hepatobiliar o yema hepática que aparece como una excrecencia del epitelio endodérmico distal a la unión del intestino anterior y medio. Esta yema hepática brota en dos localizaciones distintas: el primordio hepático y el primordio de los conductos biliares y la vesícula biliar? Para la séptima semana se produce la vacuolización, y la vesícula biliar, el conducto cístico y el árbol biliar desarrollan una luz².

La agenesia vesicular es el resultado del fracaso en el desarrollo de la parte caudal del divertículo o yema hepática durante la embriogénesis ${ }^{8}$, debido a que la vesícula biliar y el conducto cístico no se desprenden del colédoco durante la quinta semana de gestación ${ }^{6}$. Por tanto, la agenesia sigue al fracaso de la proliferación de la yema de la vesícula biliar o al fracaso de la vacuolización del primordio de la vesícula biliar².

Se ha observado que la agenesia de vesícula biliar ocurre en grupos familiares, lo que sugiere una predisposición genética?.

La mayoría de los pacientes cursan de forma asintomática, pero la afección se descubre de manera incidental cuando presentan síntomas abdominales, de los cuales hasta el $50 \%$ son inespecíficos ${ }^{7}$. Aun así, se ha descrito que el dolor en el cuadrante superior derecho del abdomen está presente en más del $90 \%$ de estos pacientes. Otros síntomas asociados son náuseas y vómitos en un $66 \%$, intolerancia a los alimentos altos en grasas en un $37 \%$, ictericia en un $35 \%$ y dispepsia en un $30 \%{ }^{3}$. Otros pacientes pueden no presentar síntomas nunca o presentarlos durante la infancia. La sintomatología y el aumento de la frecuencia de cálculos biliares se han atribuido a la discinesia biliar'; se presenta una contracción muscular retrógrada hipertónica del esfínter de Oddi que conduce a dilatación del conducto común, estasis biliar y formación de cálculos biliares ${ }^{4}$, adjudicando o relacionando esto con causas no biliares como esofagitis y duodenitis'.

Se estima que del $25 \%$ al $50 \%$ de los pacientes con agenesia de la vesícula biliar pueden desarrollar coledocolitiasis secundaria a estasis de las vías biliares $^{7}$. La ictericia se debe a una coledocolitiasis asociada con o sin colangitis ascendente'.

Los pacientes con agenesia de la vesícula biliar han sido categorizados en tres grupos dependiendo de la presentación clínica: 1) aquellos con múltiples anomalías fetales detectadas perinatalmente (15\%), 2) casos asintomáticos encontrados en la autopsia o incidentalmente durante la operación, y 3) casos sintomáticos que suelen presentarse en la tercera a cuarta décadas de la vida $(50 \%)^{8,9}$.

Se ha observado que la agenesia de la vesícula biliar ocurre en grupos familiares, lo que sugiere una predisposición genética ${ }^{10}$. A menudo se asocia con malformaciones adicionales, como defectos del tabique cardiaco, atresia duodenal, ano imperforado, divisum pancreático y agenesia renal en el 12.8-30\% de los $\operatorname{casos}^{1,8}$.

La dificultad diagnóstica radica en la incertidumbre clínica y los riesgos que se tienen si no se toma en cuenta el diagnóstico probable. La ausencia de vesícula biliar en la ultrasonografía abdominal o de hígado y vías biliares, considerada como la prueba de imagen inicial, confirma el diagnóstico; pese a esto, es importante reconocer que no todos los informes de ausencia de visualización de la vesícula biliar implican un diagnóstico de agenesia ${ }^{7}$, ya que con frecuencia se confunde con una vesícula biliar excluida 0 escleroatrófica ${ }^{4}$. Aunque la ultrasonografía abdominal o de hígado y vías biliares tiene unas altas sensibilidad y especificidad para la patología de la vesícula biliar, depende del operador y de otras variables que afectan las características de desempeño de la 
ecografía en el diagnóstico ${ }^{7}$. En este contexto, el uso apropiado de modalidades de investigación adicionales, como la tomografía computarizada, la colangiopancreatografía por resonancia magnética, la ultrasonografía y la CPRE, a menudo proporcionarán la información anatómica necesaria para hacer un diagnóstico preoperatorio y ayudar a evitar una cirugía injustificada ${ }^{7,11}$.

Si el diagnóstico se realiza durante la operación, el cirujano debe probar examinando minuciosamente los sitios más comunes de localización ectópica que están descritos en la literatura, tales como intrahepática, retrohepática, en el lado izquierdo, dentro de las hojas del epiplón menor, dentro del ligamento falciforme, retroduodenal, retropancreática y retroperitoneal ${ }^{1,12}$.

La indicación quirúrgica en los pacientes suele ser por una queja de síntomas biliares junto con un estudio de imagen falso positivo'. La mayoría de los pacientes reportados en la literatura se volvieron asintomáticos después de los procedimientos quirúrgicos. Si los síntomas continúan en el posoperatorio, el tratamiento con relajantes del músculo liso y analgésicos es eficaz ${ }^{1}$. Si a pesar de esto los síntomas persisten, entonces el paciente debe ser investigado con tomografía computarizada, manometría biliar, endoscopia digestiva alta y CPRE con o sin esfinterotomía. Rara vez se necesitan procedimientos endoscópicos de ablación del esfínter'1.

Si el diagnóstico se hace preoperatoriamente, los pacientes que tienen coledocolitiasis deben someterse a extracción de cálculos por CPRE o coledocolitotomía; de lo contrario, no se requiere tratamiento o procedimiento definitivo si no se presentan síntomas. En los pacientes con agenesia aislada y sanos, el pronóstico es excelente ${ }^{1}$. Para los pacientes sintomáticos con agenesia aislada es adecuado el tratamiento conservador, a menos que estén presentes otras enfermedades biliares ${ }^{1}$.

\section{Agradecimientos}

Los autores agradecen al servicio de cirugía general del Hospital General de Puebla, por prestar todas las facilidades para poder llevar a cabo esta publicación, y al Dr. Manuel Gil Vargas por su asesoría.

\section{Financiamiento}

Los autores no cuentan con financiamiento de ningún tipo.

\section{Conflicto de intereses}

Los autores no cuentan con conflicto de intereses de ningún tipo.

\section{Responsabilidades éticas}

Protección de personas y animales. Los autores declaran que para esta investigación no se han realizado experimentos en seres humanos ni en animales.

Confidencialidad de los datos. Los autores declaran que en este artículo no aparecen datos de pacientes.

Derecho a la privacidad y consentimiento informado. Los autores han obtenido el consentimiento informado de los pacientes y/o sujetos referidos en el artículo. Este documento obra en poder del autor de correspondencia.

\section{Bibliografía}

1. Bani-Hani KE. Agenesis of the gallbladder: difficulties in management. J Gastroenterol Hepatol. 2005;20:671-5.

2. Kabiri $\mathrm{H}$, Domingo $\mathrm{OH}$, Tzarnas $\mathrm{CD}$. Agenesis of the gallbladder. Curr Surg. 2006;63:104-6.

3. Gotohda N, Itano S, Horiki S, Endo A, Nakao A, Terada N, et al. Gallbladder agenesis with no other biliary tract abnormality: report of a case and review of the literature. $J$ Hepatobiliary Pancreat Surg. 2000;7:327-30

4. Peloponissios N, Gillet M, Cavin R, Halkic N. Agenesis of the gallbladder: a dangerously misdiagnosed malformation. World $\mathrm{J}$ Gastroenterol. 2005;11:6228-31.

5. Aneiros Castro B, Cano Novillo I, García Vázquez A, De Miguel Moya M. Association between gallbladder agenesis and choledochal cyst: cause or coincidence? BMJ Case Rep. 2019;12:12-4.

6. Kasi PM, Ramirez R, Rogal SS, Littleton K, Fasanella KE. Gallbladder agenesis. Case Rep Gastroenterol. 2011;5:654-62.

7. Bedi N, Bond-Smith G, Kumar S, Hutchins R. Gallbladder agenesis with choledochal cyst - a rare association: a case report and review of possible genetic or embryological links. BMJ Case Rep. 2013;2013:bcr2012006786.

8. Rajkumar A, Piya A. Gall bladder agenesis: a rare embryonic cause of recurrent biliary colic. Am J Case Rep. 2017;18:334-8.

9. Calder N, Carneiro HA, Khwaja HA, Thompson JN. Gallbladder agenesis with midgut malrotation. BMJ Case Rep. 2012;2012:bcr1020115053.

10. Yoldas O, Yazıcı P, Ozsan I, Karabuga T, Alpdogan O, Sahin E, et al. Coexistence of gallbladder agenesis and cholangiocarcinoma: report of a case. J Gastrointest Surg. 2014;18:1373-6.

11. Yener O, Buldanlı MZ, Eksioglu H, Leblebici M, Alimoglu O. Agenesis of the gallbladder diagnosed by magnetic resonance cholangio graphy: report of a case and review of the literature. Prague Med Rep. 2015;116:52-6.

12. Salazar MC, Brownson KE, Nadzam GS, Duffy A, Roberts KE. Gallbladder agenesis: a case report. Yale J Biol Med. 2018;91:237-41. 